\title{
Evaluation of Wheat Genotypes for Protective Mechanisms of Terminal Heat Stress
}

\author{
Pooja $^{1}$, Renu Munjal ${ }^{2}$ and Kirpa Ram ${ }^{3}$ \\ ${ }^{1}$ Department of Botany and Plant Physiology, ${ }^{2}$ Department of Genetics and Plant Breeding, CCS \\ Haryana Agricultural University, Hisar 125004, Haryana, India \\ *Corresponding author
}

A B S T R A C T

Keywords

Wheat, Terminal heat stress, MDA content and cell membrane stability.

Article Info

Accepted:

20 September 2017

Available Online:

10 November 2017
Wheat is the one of the most important cereal crop in the world. High temperature stress is a common environmental phenomena encounter by wheat throughout the world. Wheat is sensitive to high temperature; increase in temperature is a severe threat to wheat production and quality loss, particularly when it occurs during reproductive and grainfilling phases. Keeping in view a field experiment was conducted to evaluate twenty wheat genotypes (ten heat tolerant and ten heat susceptible) for various physiological, biochemical traits at anthesis, seven days after anthesis and identify promising wheat genotypes under terminal high temperature condition. High temperature significantly influenced physiological, biochemical, yield and yield attributes at anthesis and 14 days after anthesis (DAA) in both heat susceptible and heat tolerant genotypes. Decrease in carotenoids content and cell membrane stability (CMS) was least in heat tolerant genotype i.e., WH 730 (9.4\%) and WH 1021 (10.5\%) respectively. WH 1021 showed high cell membrane stability (CMS), carotenoids content at anthesis and 7DAA. WH 1021 also, retained highest biomass, grain yield and 1000 grain weight. In present investigation heat tolerant genotype WH 1021 was found to be the most suitable one terminal high temperature condition on the basis of the overall picture of physiological, biochemical and vield traits studied

\section{Introduction}

Wheat is the single most important crop on global scale in terms of total harvested weight and amount used for human and animal nutrition. It is grown across an exceptionally diverse range of environments, from the arid plains of Africa to the humid valleys of Vietnam and from the cold of Nepal to the heat of India Rao et al., (1977). Currently in India, wheat is grown on an area of about 30.37 million hectares which produces 90.78 million tons of wheat with a productivity of 2.99 tons per hectare making India second largest producer of wheat in the world (Anonymous, 2015). In India wheat production is hindered by various factors like date of sowing, judicious application of fertilizers, irrigation time and temperature. Out of these, high temperature is a crucial environmental aspect (Slafer and Rawson, 1994). High temperature stress induces oxidative stress. Protective responses must be triggered quickly in response to the oxidative stress effectors to prevent plant from damage. Studies have shown that protection 
mechanisms like anti-oxidative compounds, which helped to prevent the accumulation of reactive oxidant species (ROS), membrane lipid peroxidation and maintenance high cell membrane stability play an important role. Membrane is the first line of defense having many heat responsive sensors which helps plant to trigger its defense mechanism against heat stress, so the integrity of the membrane is an important parameter for heat tolerance. Cell membrane stability (CMS) is directly correlated with outside environmental temperature and leaf electrolyte leakage, as a measure of leaf membrane thermostability, may provide an efficient indirect screening technique for reproductive-stage heattolerance genes. High temperature stress induces intense changes in cellular membranes which results in loss of membrane stability index (MSI) resulting in ion/solute leakage Saxena et al., (2016). Heat stress triggers the production and accumulation of ROS Sairam et al., (2000); Mittler (2002); Almeselmani et al., (2009) which causes oxidative stress may induce lipid peroxidation leading to protein degradation, membrane rupture and enzyme inactivation Sairam et al., (2000). Lipid peroxidation is considered as one of the most damaging processes.

MDA content reflects the degree of damage at adverse conditions and is an indicator of lipid peroxidation. Mishra et al., (2017) who reported 3 fold increases in MDA in wheat under heat stress. Dhyani et al., (2013) also who reported more MDA content at anthesis and 15 days after anthesis under terminal heat stress. Sairam et al., (2000).Detoxification of ROS produced in oxidative stress caused by heat stress by antioxidant systems is important for protecting plants against heat stress (Asada, 2006). The capacity of the cellular anti oxidative and photo protective defense is determined by the pool size of antioxidants and protective pigments Karin et al., (2002). Carotenoids (Car) are necessary for photoprotection of photosynthesis and they play an important role as a precursor in signaling during the plant development under abiotic/biotic stress. Carotenoids protect chlorophyll from photo oxidative destruction, so a change in carotenoids could have serious consequences for the effect of UV-B radiation on chlorophyll pigments Mishra et al., (2008). Physiological and Biochemical parameters like CMS, Lipid peroxidation-MDA content and Total antioxidant activity (TAA) and carotenoids content might be helpful to overcome yield loss under terminal heat stress. Keeping in view study was conducted to evaluate various physiological, biochemical traits and to identify a promising wheat genotype under terminal high temperature condition.

\section{Materials and Methods}

The twenty wheat genotypes i.e. ten tolerant (WH 730, WH 1124, WH 1021, HD 3059, DBW 90, PBW 373, Raj 3765, HD 2851, HD 2285, PBW 550) and ten heat susceptible (HD 2967, DBW 621, WH 1105, DBW 88, HD 3086, HD 2733, WH 711, WH 1080, WH 1142 and $\mathrm{K}$ 0307)were evaluated under terminal high temperature condition. The crop was sown in middle of December, 2016 (late planting date). The experiments were carried out through a randomized block design consisting of 4 rows of $3 \mathrm{~m}$ length with $20 \times 5$ $\mathrm{cm}$ spacing within rows and between plants, respectively with three replications in field crop research area of Wheat and Barley Section, Department of Genetics and Plant Breeding, CCS HAU, Hisar India.

Flag leaf per selected plant was randomly chosen and tagged (total of ten flag leaves per genotype was tagged). The tagged leaves were used to measure physiological and biochemical traits (cell membrane stability, lipid peroxidation-MDA content, total antioxidant activity and carotenoids content) at anthesis and 14 days after anthesis (14DAA). 


\section{Cell membrane stability (\%)}

To measure cell membrane stability, method of Sullivan (1972), modified later on by Ibrahim and Quick (2001) was followed. A random sample of flag leaf from 3 plants from each replication was collected from anthesis till maturity at interval of seven days. Each sample was collected in sealed plastic bags and immediately kept in ice box. At laboratory all the samples were thoroughly rinsed twice in deionized water. The mid rib of flag leaves was removed gently by hand and about $5 \mathrm{~cm}$ portion from central flag leaf area was excised and cut in to 4 equal parts. Leaf was taken in glass test tubes containing $10 \mathrm{ml}$ distilled water. The test tube samples were tightly covered with aluminum foil and were maintained between $4-8{ }^{\circ} \mathrm{C}$ for $24 \mathrm{hrs}$, and after that, the test tubes were kept at room temperature and reading 1 was measured. After that tubes were heated to $49^{\circ} \mathrm{C}$ in the water-bath for exposing the leaf samples for 1 hour. Then, again leaf samples were maintained at $4-8^{\circ} \mathrm{C}$ for $24 \mathrm{hrs}$, and then samples were brought to room temperature, reading 2 was taken. Finally, tubes were autoclaved for $30 \mathrm{~min}$ at $121^{\circ} \mathrm{C}$ and then tubes were again kept at $4-8^{\circ} \mathrm{C}$ for $24 \mathrm{hrs}$, which were brought to room temperature for taking reading 3 .

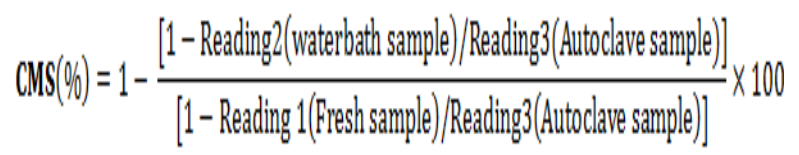

\section{Lipid peroxidation-MDA content ( $\mu$ mole/g fresh weight)}

Malondialdehyde (MDA) content was estimated according to the method of Heath and Packer (1968), where approximately $0.5 \mathrm{~g}$ leaf sample was homogenized in $5 \mathrm{ml}$ of $0.1 \%$ trichloroacetic acid (TCA) with the help of mortar and pestle and centrifuged at 8,000 $\mathrm{rpm}$ for $15 \mathrm{~min}$. After centrifugation, $1 \mathrm{ml}$ of the supernatant was mixed with $2.3 \mathrm{ml} 0.5 \%$ TBA in 20\% TCA and incubated in hot water $\left(95^{\circ} \mathrm{C}\right)$ for $30 \mathrm{~min}$. Thereafter, it was cooled immediately on ice to stop the reaction. Absorbance at 532 and $600 \mathrm{~nm}$ was determined, and MDA concentration was estimated by subtracting the non-specific absorption at $600 \mathrm{~nm}$ from the absorption at $532 \mathrm{~nm}$, using an absorbance coefficient of extinction $\left(155 \mathrm{mM}^{-1} \mathrm{~cm}^{-1}\right)$.

\section{Total antioxidant activity ( $\mu$ mole/g fresh weight)}

Total antioxidant activity was estimated by the method of Prieto et al., (1999) where Sample material of $0.5 \mathrm{~g}$ was homogenized in $5 \mathrm{ml}$ of $95 \%$ methanol, contents was transferred into $150 \mathrm{ml}$ conical flasks. Conical flask was sealed with parafilm, kept on shaker for one hour, centrifuged the contents at $10,000 \mathrm{rpm}$ for 20 minutes, transferred and the supernatant was used for estimation.

Transferred $0.3 \mathrm{ml}$ of supernatant, $3 \mathrm{ml}$ of phosphomolybdate reagent into polycarbon capped tubes, incubated the contents on water bath at $95^{\circ} \mathrm{C}$ for 90 minutes, cooled the contents to room temperature and absorbance was read against blank at $695 \mathrm{~nm}$. Total antioxidant activity was estimated from standard curve of ascorbic acid (10-100 $\mu \mathrm{g})$ and expressed in terms of ascorbic acid equivalent.

\section{Carotenoids content ( $\left.\mathrm{mg} \mathrm{g}^{-1}\right)$}

Carotenoids content was estimated by the method of Hiscox and Isrealstam (1979), where $100 \mathrm{mg}$ washed and finely chopped leaves' (excluding veins) was placed in a tube containing $5 \mathrm{ml}$ DMSO.

These tubes were incubated at $65^{\circ} \mathrm{C}$ for one hour. Extract was transferred to a $10 \mathrm{ml}$ graduated cylinder and volume was made up 
to mark with DMSO. Absorbance was measured at $450 \mathrm{~nm}$. Cartenoids content was calculated by following formula:

Carotenoids content $=\frac{10 \times \mathrm{A} 450 \times \mathrm{V}}{2500 \times \mathrm{W}}$

$\mathrm{V}=$ Volume of the solution used / Dilution factor

$\mathrm{W}=$ Weight of the sample

Yield and yield attributes

\section{Biomass $\left(\mathrm{kg} / \mathrm{m}^{2}\right)$}

Plants were cut from the base of stem at maturity and weight was taken using spring balance in kilograms and average was taken.

\section{Grain yield $\left(\mathrm{kg} / \mathrm{m}^{2}\right)$}

Grain yield from each plot was recorded in kilograms.

\section{Thousand grain weight (g)}

Weight of randomly chosen clean and filled 1000 grains was measured in grams from each replication using electronic balance and average was recorded.

\section{Harvest index (\%)}

Harvest index for each of the genotype was computed using the following formula;

Harvest Index $(\%)=\frac{\text { Economic yield }}{\text { Biological yield }} \times 100$

\section{Statistical analysis}

Statistical analysis was done using Indian NARS Statistical Computing Portal: http:// stat.iasri.res.in/sscnarsportal of IASRI New Delhi.

\section{Results and Discussion}

\section{Physiological and biochemical traits}

Significant differences existed among wheat genotypes tested for total antioxidant activity (TAA), cell membrane stability (CMS), lipid peroxidation in term of MDA content and carotenoids content at the anthesis and 14DAA stages (Table 1). At anthesis TAA ranged from $11.09 \mu$ mole/g fresh weight in $\mathrm{K}$ 0307 to $66.95 \mu$ mole/g fresh weight. In WH 1124 same trend was observed at 14DAA. CMS decreased from anthesis to 14DAA. At anthesis CMS ranged from 54.54\% in $\mathrm{K} 0307$ to $75.16 \%$ in WH 1021 also $14 \mathrm{DAA}$ it ranged from $23.84 \%$ in $\mathrm{K} 0307$ to $67.29 \%$ in $\mathrm{WH}$ 1021. The Lipid peroxidation was measured in terms of MDA content. MDA content ranged from $2.71 \mu \mathrm{mole} / \mathrm{g}$ fresh weight in PBW373 to 9.10umole/g fresh in HD 2967 at anthesis whereas, at 14DAA MDA content ranged from $7.10 \mu$ mole/g fresh weight in HD 3059 to $12.19 \mu \mathrm{mole} / \mathrm{g}$ fresh weight in WH711. Carotenoids content decreased from anthesis to 14DAA. At anthesis carotenoids content ranged from $0.49 \mathrm{mg} \mathrm{g}^{-1}$ in $\mathrm{K} 0307$ to $1.07 \mathrm{mg} \mathrm{g}^{-1}$ in WH 1021 whereas it ranged from $0.35 \mathrm{mg} \mathrm{g}^{-1}$ in $\mathrm{K} 0307$ to $0.69 \mathrm{mg} \mathrm{g}^{-1}$ in WH 1021 14DAA. Average TAA, CMS, lipid peroxidation-MDA content, Carotenoids content was 48.88, 66.86, 6.52, 0.79 respectively at anthesis and 66.74, 40.30, 9.66 and 0.52 respectively at 14DAA which indicates significant effect of heat stress at anthesis and 14DAA.

WH 730 (9.4 \%) and WH 1021 (10.5\%) showed minimum decrease in carotenoids content and CMS respectively whereas WH $1080(56.1 \%)$ and K 0307 (56.3\%) showed maximum decrease for Carotenoids content and CMS respectively. PBW $373(60 \%)$ and HD 2967 (69.8\%) showed maximum increase for lipid peroxidation - MDA content and total antioxidant activity whereas K 0307 
(23.0\%) and HD 3059 (13.0\%) showed for minimum increase for lipid peroxidation MDA content and TAA respectively (Table 2).

Relation between cell membrane stability and total antioxidant activity, lipid peroxidation, carotenoids content under terminal heat stress condition

The results pertaining to relation between cell membrane stability and total antioxidant activity, CMS and lipid peroxidation - MDA content, CMS and carotenoids is shown in Figures 1, 2 and 3. A positive relation was found between CMS and TAA for all the genotypes. A positive relation between CMS and carotenoids content was observed in all genotypes. But negative relation between CMS and lipid peroxidation was observed in few genotypes (HD 3059, PBW 550, WH1124, WH 1021). TAA, carotenoids content and less lipid peroxidation could be possible reason for high cell membrane stability.

Table.1 Various physiological and biochemical traits under terminal heat stress condition

\begin{tabular}{|c|c|c|c|c|c|c|c|c|}
\hline \multirow[t]{2}{*}{ Genotypes } & \multicolumn{2}{|c|}{$\begin{array}{c}\text { Total antioxidant } \\
\text { activity(TAA) }\end{array}$} & \multicolumn{2}{|c|}{$\begin{array}{l}\text { Cell membrane } \\
\text { stability (CMS) }\end{array}$} & \multicolumn{2}{|c|}{$\begin{array}{l}\text { Lipid peroxidation- } \\
\text { MDA content }\end{array}$} & \multicolumn{2}{|c|}{$\begin{array}{c}\text { Carotenoids } \\
\text { content }\end{array}$} \\
\hline & Anthesis & 14DAA & Anthesis & 14DAA & Anthesis & 14DAA & Anthesis & 14DAA \\
\hline DBW621 & $45.10^{\mathrm{H}}$ & $62.55^{\mathrm{IJ}}$ & $61.52^{\mathrm{I}}$ & $30.03^{\mathrm{JK}}$ & $7.55^{\mathrm{CD}}$ & $10.61^{\mathrm{BCD}}$ & $0.66^{\mathrm{K}}$ & $0.42^{\mathrm{JK}}$ \\
\hline DBW88 & $49.80^{\mathrm{FGH}}$ & $64.48^{\mathrm{HI}}$ & $64.05^{\mathrm{H}}$ & $32.24^{\mathrm{IJ}}$ & $7.05^{\mathrm{EF}}$ & $10.19^{\mathrm{CDE}}$ & $0.68^{\mathrm{IJK}}$ & $0.45^{\mathrm{IJK}}$ \\
\hline DBW90 & $58.41^{\mathrm{BCD}}$ & $71.01^{\mathrm{BCDE}}$ & $71.46^{\mathrm{BC}}$ & $47.61^{\mathrm{D}}$ & $5.85^{\mathrm{L}}$ & $8.95^{\mathrm{IJ}}$ & $0.91^{\mathrm{CDE}}$ & $0.59^{\mathrm{DE}}$ \\
\hline HD2285 & $52.29^{\mathrm{EFG}}$ & $68.55^{\mathrm{DEFG}}$ & $68.75^{\mathrm{DEF}}$ & $41.78^{\mathrm{F}}$ & $6.42^{\mathrm{HIJ}}$ & $9.48^{\text {FGHI }}$ & $0.80^{\mathrm{FG}}$ & $0.55^{\mathrm{EFG}}$ \\
\hline HD2733 & $47.32^{\mathrm{GH}}$ & $63.07^{\mathrm{IJ}}$ & $61.67^{\mathrm{I}}$ & $31.45^{\mathrm{IJ}}$ & $7.32^{\mathrm{DE}}$ & $10.26^{\mathrm{CDE}}$ & $0.68^{\mathrm{JK}}$ & $0.43^{\mathrm{JK}}$ \\
\hline HD2851 & $52.00^{\mathrm{EFG}}$ & $68.46^{\mathrm{EFG}}$ & $68.06^{\mathrm{EFG}}$ & $39.32^{\mathrm{FG}}$ & $6.53^{\mathrm{GHI}}$ & $9.76^{\mathrm{EFGH}}$ & $0.78^{\mathrm{GH}}$ & $0.54^{\mathrm{EFG}}$ \\
\hline HD2967 & $18.21^{\mathrm{J}}$ & $60.35^{\mathrm{J}}$ & $56.21^{\mathrm{K}}$ & $27.11^{\mathrm{KL}}$ & $9.10^{A}$ & $11.19^{\mathrm{B}}$ & $0.58^{\mathrm{L}}$ & $0.38^{\mathrm{LM}}$ \\
\hline HD3059 & $63.31^{\mathrm{AB}}$ & $71.57^{\mathrm{BC}}$ & $73.69^{\mathrm{AB}}$ & $53.44^{\mathrm{C}}$ & $5.47^{\mathrm{MN}}$ & $7.10^{\mathrm{M}}$ & $0.97^{\mathrm{BC}}$ & $0.64^{\mathrm{BC}}$ \\
\hline HD3086 & $50.97^{\mathrm{EFG}}$ & $68.17^{\mathrm{EFG}}$ & $67.78^{\mathrm{EFG}}$ & $38.28^{\mathrm{G}}$ & $6.58^{\mathrm{GHI}}$ & $9.90^{\mathrm{EFG}}$ & $0.75^{\mathrm{GHI}}$ & $0.52^{\mathrm{FGH}}$ \\
\hline K0307 & $11.09^{\mathrm{K}}$ & $23.09^{K}$ & $54.54^{\mathrm{K}}$ & $23.84^{\mathrm{L}}$ & $8.79^{\mathrm{B}}$ & $10.81^{\mathrm{BC}}$ & $0.49^{\mathrm{M}}$ & $\mathbf{0 . 3 5} 5^{\mathrm{M}}$ \\
\hline PBW373 & $53.60^{\mathrm{DEF}}$ & $69.17^{\mathrm{CDEFG}}$ & $69.53^{\mathrm{CDE}}$ & $42.21^{\mathrm{EF}}$ & $2.71^{O}$ & $9.37^{\mathrm{GHIJ}}$ & $0.85^{\mathrm{EF}}$ & $0.56^{\mathrm{EF}}$ \\
\hline PBW550 & $63.62^{\mathrm{AB}}$ & $72.24^{\mathrm{B}}$ & $74.58^{\mathrm{A}}$ & $57.55^{\mathrm{B}}$ & $5.19^{\mathrm{N}}$ & $8.29^{\mathrm{KL}}$ & $1.01^{\mathrm{AB}}$ & $0.68^{\mathrm{AB}}$ \\
\hline Raj3765 & $56.04^{\mathrm{CDE}}$ & $70.32^{\text {BCDEF }}$ & $70.48^{\mathrm{CD}}$ & $45.40^{\mathrm{DE}}$ & $5.98^{\mathrm{KL}}$ & $9.13^{\mathrm{HIJ}}$ & $0.90^{\mathrm{DE}}$ & $0.58^{\mathrm{DE}}$ \\
\hline WH1021 & $59.66^{\mathrm{BC}}$ & $71.50^{\mathrm{BCD}}$ & $75.16^{A}$ & $67.29^{A}$ & $6.29^{\mathrm{IJ}}$ & $7.79^{\mathrm{L}}$ & $1.07^{\mathrm{A}}$ & $0.69^{A}$ \\
\hline WH1080 & $50.06^{\mathrm{FGH}}$ & $66.28^{\mathrm{GH}}$ & $64.40^{\mathrm{H}}$ & $32.94^{\mathrm{IJ}}$ & $6.81^{\mathrm{FG}}$ & $10.13^{\mathrm{DE}}$ & $0.70^{\mathrm{IJK}}$ & $0.47^{\mathrm{HIJ}}$ \\
\hline WH1105 & $50.42^{\text {FGH }}$ & $66.69^{\mathrm{GH}}$ & $66.07^{\mathrm{GH}}$ & $34.72^{\mathrm{HI}}$ & $6.73^{\mathrm{G}}$ & $10.05^{\mathrm{DEF}}$ & $0.71^{\mathrm{HIJK}}$ & $0.48^{\mathrm{HI}}$ \\
\hline WH1124 & $66.95^{\mathrm{A}}$ & $76.89^{A}$ & $73.27^{\mathrm{AB}}$ & $51.49^{\mathrm{C}}$ & $5.56^{\mathrm{M}}$ & $8.81^{\mathrm{JK}}$ & $0.95^{\mathrm{BCD}}$ & $0.62^{\mathrm{CD}}$ \\
\hline WH1142 & $50.42^{\mathrm{FGH}}$ & $67.31^{\mathrm{FGH}}$ & $67.07^{\mathrm{FG}}$ & $37.32^{\mathrm{GH}}$ & $6.64^{\mathrm{GH}}$ & $9.95^{\mathrm{EFG}}$ & $0.74^{\mathrm{GHIJ}}$ & $0.50^{\mathrm{GH}}$ \\
\hline WH711 & $23.72^{\mathrm{I}}$ & $61.83^{\mathrm{IJ}}$ & $59.06^{\mathrm{J}}$ & $29.60^{\mathrm{JK}}$ & $7.63^{\mathrm{C}}$ & $12.19^{A}$ & $0.64^{\mathrm{KL}}$ & $0.41^{\mathrm{KL}}$ \\
\hline WH730 & $54.58^{\mathrm{CDEF}}$ & $69.81^{\text {BCDEF }}$ & $69.93^{\mathrm{CDE}}$ & $42.32^{\mathrm{EF}}$ & $6.24^{\mathrm{JK}}$ & $9.32^{\mathrm{GHIJ}}$ & $0.86^{\mathrm{EF}}$ & $0.58^{\mathrm{DE}}$ \\
\hline Mean & 48.88 & 66.74 & 66.86 & 40.3 & 6.52 & 9.66 & 0.79 & 0.52 \\
\hline C.V (\%) & 5.23 & 2.16 & 1.61 & 4.03 & 2.11 & 3.17 & 4.35 & 9.45 \\
\hline
\end{tabular}

*Mean values followed by the same letters in TAA, CMS, lipid peroxidation -MDA and carotenoid content columns are not significantly different at $5 \%$ level of probability according to Duncan's multiple range test 
Table.2 Increase or decrease in various physiological and biochemical traits in wheat genotypes from anthesis to 14DAA under terminal heat stress condition

\begin{tabular}{|c|c|c|c|c|}
\hline \multirow{2}{*}{ Genotypes } & \multicolumn{2}{|c|}{$\%$ Increase } & \multicolumn{2}{|c|}{$\%$ Decrease } \\
\hline & TAA & MDA & CAR & CMS \\
\hline DBW 621 & 38.7 & 40.5 & 36.4 & 51.2 \\
\hline DBW88 & 29.5 & 44.5 & 33.8 & 49.7 \\
\hline DBW90 & 21.6 & 53.0 & 35.2 & 33.4 \\
\hline HD2285 & 31.1 & 47.7 & 31.3 & 39.2 \\
\hline HD2733 & 33.3 & 40.2 & 36.8 & 49.0 \\
\hline HD2851 & 31.7 & 49.5 & 30.8 & 42.2 \\
\hline HD2967 & 69.8 & 23.0 & 34.5 & 51.8 \\
\hline HD3059 & 13.0 & 29.8 & 34.0 & 27.5 \\
\hline HD3086 & 33.7 & 50.5 & 30.7 & 43.5 \\
\hline K0307 & 52.0 & 23.0 & 28.6 & 56.3 \\
\hline PBW373 & 29.0 & 60.0 & 34.1 & 39.3 \\
\hline PBW550 & 13.5 & 50.1 & 32.7 & 22.8 \\
\hline Raj3765 & 25.5 & 52.7 & 35.6 & 35.6 \\
\hline WH1021 & 19.8 & 31.8 & 19.8 & 10.5 \\
\hline WH1080 & 32.4 & 48.8 & 56.1 & 48.9 \\
\hline WH1105 & 32.3 & 49.3 & 31.4 & 47.4 \\
\hline WH1124 & 14.8 & 58.5 & 12.7 & 29.7 \\
\hline WH1142 & 33.5 & 49.8 & 47.4 & 44.4 \\
\hline WH711 & 61.6 & 59.8 & 44.6 & 49.9 \\
\hline WH730 & 27.9 & 49.4 & 9.4 & 39.5 \\
\hline Range & $13.0-69.8$ & $23.0-60.0$ & $9.4-56.1$ & $10.5-56.3$ \\
\hline
\end{tabular}

TAA-Total antioxidant activity, MDA-Malondialdehyde content, CAR-Carotenoids content, CMS-Cell membrane stability 
Table.3 Yield and Yield Attributes under terminal heat stress condition

\begin{tabular}{|c|c|c|c|c|}
\hline Genotypes & Biomass $\left(\mathrm{kg} / \mathrm{m}^{2}\right)$ & Grain yield $\left(\mathrm{kg} / \mathrm{m}^{2}\right)$ & 1000 grain weight (g) & Harvest index (\%) \\
\hline DBW 621 & $0.24^{\mathrm{JKL}}$ & $0.24^{\mathrm{JKL}}$ & $27.4^{\mathrm{ABC}}$ & $34.70^{\mathrm{DE}}$ \\
\hline DBW 88 & $0.82^{\mathrm{DEF}}$ & $0.25^{\mathrm{FG}}$ & $29.1^{\mathrm{ABCDE}}$ & $34.30^{\mathrm{DE}}$ \\
\hline DBW 90 & $0.96^{\mathrm{BCDE}}$ & $0.35^{\mathrm{BCDEF}}$ & $39.9^{\mathrm{A}}$ & $34.30^{\mathrm{ABCDE}}$ \\
\hline HD 2285 & $0.94^{\mathrm{BCDE}}$ & $0.28^{\mathrm{EF}}$ & $34.0^{\mathrm{DEFG}}$ & $35.00^{\mathrm{DE}}$ \\
\hline HD 2733 & $0.84^{\mathrm{DE}}$ & $0.24^{\mathrm{FG}}$ & $28.0^{\mathbf{H}}$ & $34.00^{\mathrm{DE}}$ \\
\hline HD 2851 & $0.71^{\mathrm{EF}}$ & $0.28^{\mathrm{EF}}$ & $33.0^{\mathrm{BCDEFG}}$ & 33.30 $^{\mathrm{ABCD}}$ \\
\hline HD 2967 & $0.67^{\mathrm{EF}}$ & $0.22^{\mathrm{DEF}}$ & $22.5^{\mathrm{AB}}$ & $36.70^{\mathrm{ABCDE}}$ \\
\hline HD 3059 & $1.48^{\mathrm{A}}$ & $0.43^{\mathrm{AB}}$ & $37.7^{\mathrm{ABCDE}}$ & $\mathbf{3 7 . 3 0}^{\mathrm{DE}}$ \\
\hline HD 3086 & $0.78^{\mathrm{EF}}$ & $0.33^{\mathrm{BCDEF}}$ & $31.9^{\mathbf{A}}$ & $35.00^{\mathrm{ABCD}}$ \\
\hline К 0307 & $0.50^{\mathrm{F}}$ & $0.16^{\mathrm{G}}$ & $\mathbf{1 8 . 5}^{\mathrm{EFGH}}$ & $33.70^{\mathrm{CDE}}$ \\
\hline PBW 373 & $0.93^{\mathrm{BCDE}}$ & $0.32^{\mathrm{CDEF}}$ & $34.5^{\mathrm{FGH}}$ & $36.30^{\mathrm{CDE}}$ \\
\hline PBW 550 & $1.12^{\mathrm{BCD}}$ & $0.48^{\mathrm{BCD}}$ & $36.3^{\mathrm{BCDEF}}$ & $36.70^{\mathrm{ABC}}$ \\
\hline Raj 3765 & $0.86^{\mathrm{CDE}}$ & $0.42^{\mathrm{ABC}}$ & $35.9^{\mathrm{CDEFG}}$ & $35.00^{\mathrm{A}}$ \\
\hline WH 1021 & $1.19^{\mathrm{AB}}$ & $\mathbf{0 . 5 3}^{\mathrm{A}}$ & 40.5 . $^{\text {BCDEF }}$ & $35.00^{\mathrm{BCDE}}$ \\
\hline WH 1080 & $0.96^{\mathrm{BCDE}}$ & $0.31^{\mathrm{DEF}}$ & $29.8^{\mathrm{ABCDE}}$ & $34.70^{\mathrm{CDE}}$ \\
\hline WH 1105 & $0.88^{\mathrm{BCDE}}$ & $0.28^{\mathrm{EF}}$ & $31.1^{\mathrm{GH}}$ & $35.30^{\mathrm{CDE}}$ \\
\hline WH 1124 & $0.92^{\mathrm{BCDE}}$ & $0.39^{\mathrm{EFG}}$ & $39.3^{\mathrm{ABCDE}}$ & $34.30^{\mathrm{DE}}$ \\
\hline WH 1142 & $0.76^{\mathrm{EF}}$ & $0.37^{\mathrm{BCDE}}$ & $31.5^{\mathrm{ABCD}}$ & $34.70^{\mathrm{AB}}$ \\
\hline WH 711 & $0.80^{\mathrm{DEF}}$ & $0.21^{\mathrm{FG}}$ & $25.5^{\mathrm{ABCDE}}$ & $34.00^{\mathrm{ABCDE}}$ \\
\hline WH 730 & $1.18^{\mathrm{ABC}}$ & $0.29^{\mathrm{EF}}$ & $35.5^{\mathrm{A}}$ & $35.00^{\mathrm{E}}$ \\
\hline Mean & 0.91 & 0.32 & 35.0 & 34.96 \\
\hline LSD at $5 \%$ & 0.12 & 0.04 & 9.68 & NS \\
\hline CV (\%) & 8.34 & 8.48 & 16.27 & 5.74 \\
\hline
\end{tabular}

*Mean values followed by the same letters in Biomass, Grain yield, 1000 grain weight and Harvest Index columns are not significantly different at $5 \%$ level of probability according to Duncan's multiple range

Yield and Yield Attributes under terminal heat stress condition

Fig.1 Relation between CMS and total antioxidant activity in wheat genotypes under terminal heat stress condition

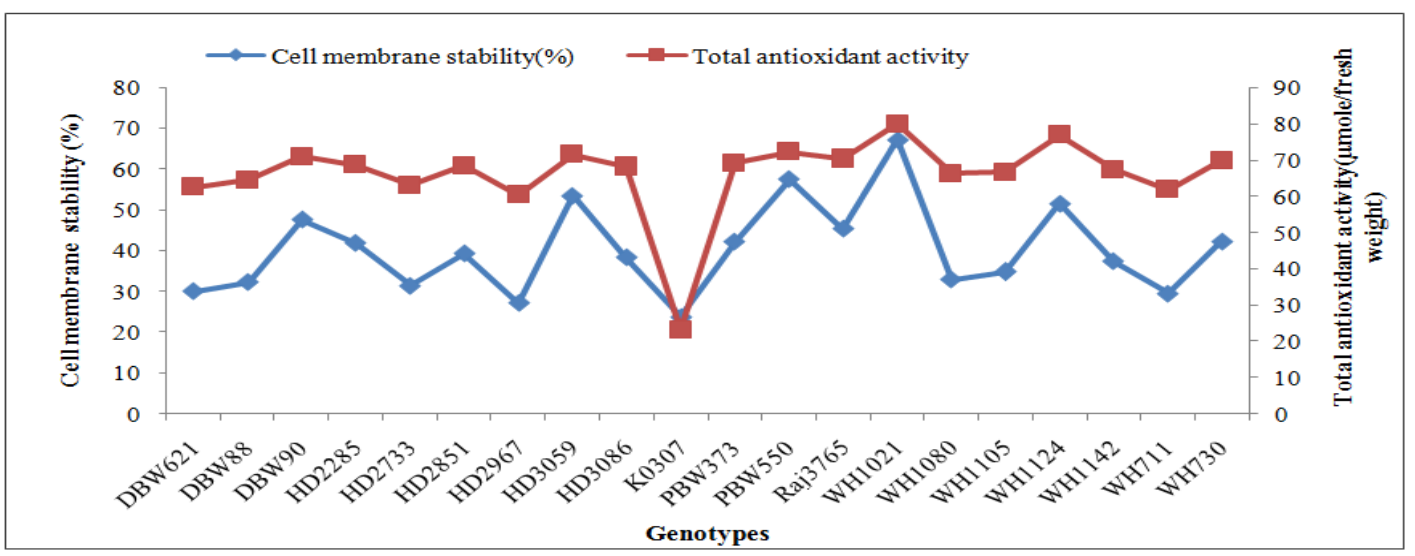


Fig.2 Relation between CMS and lipid peroxidation - MDA content in wheat genotypes under terminal heat stress condition

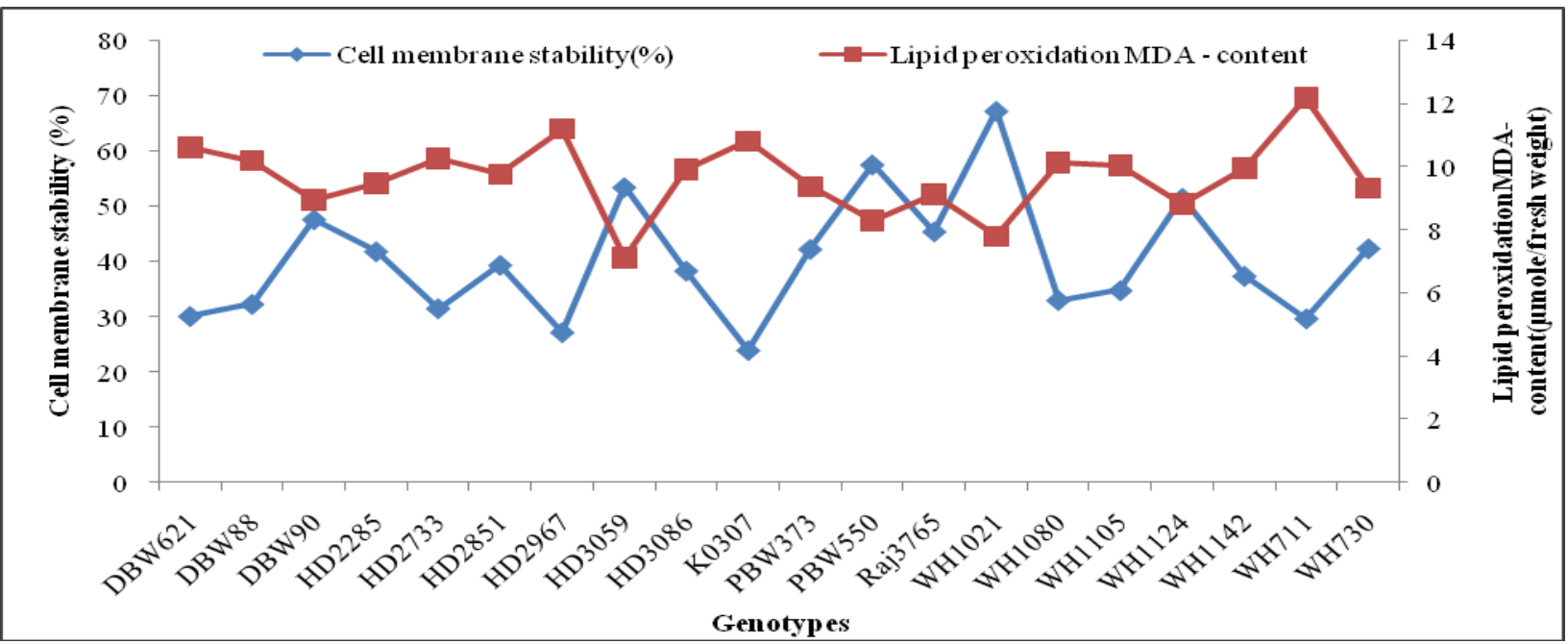

Fig.3 Relation between CMS and carotenoids content in wheat genotypes under terminal heat stress condition

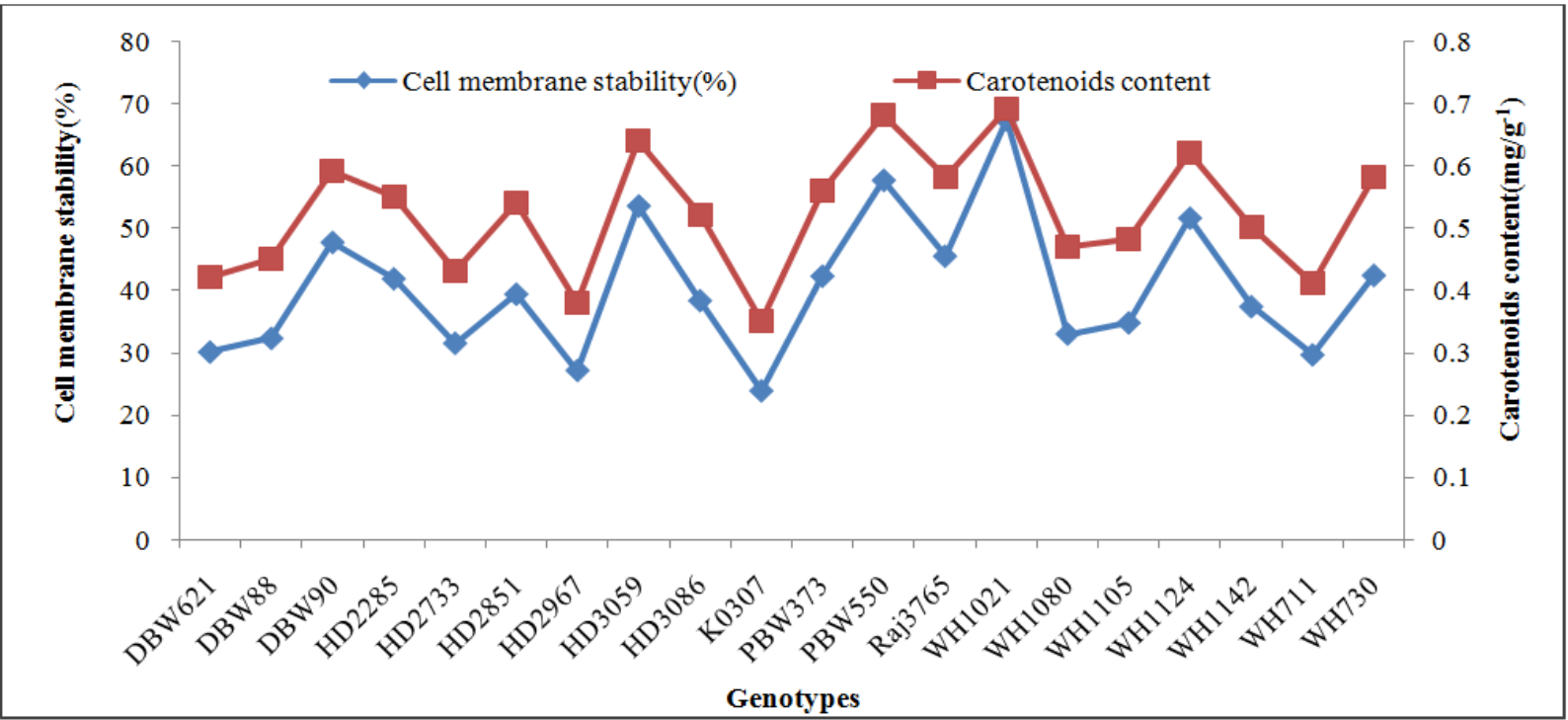

Yield and Yield Attributes

Highest biomass $\left(\mathrm{kg} / \mathrm{m}^{2}\right)$ was found in $\mathrm{WH}$ $1021\left(1.19 \mathrm{~kg} / \mathrm{m}^{2}\right)$ followed by PBW 550 $\left(1.12 \mathrm{~kg} / \mathrm{m}^{2}\right)$ and HD $2851\left(0.71 \mathrm{~kg} / \mathrm{m}^{2}\right)$. Lowest biomass $\left(\mathrm{kg} / \mathrm{m}^{2}\right)$ was found in $\mathrm{K} 0307$ $\left(0.50 \mathrm{~kg} / \mathrm{m}^{2}\right)$ followed by WH 711 $\left(0.80 \mathrm{~kg} / \mathrm{m}^{2}\right)$, WH $1142\left(0.76 \mathrm{~kg} / \mathrm{m}^{2}\right)$. Grain yield $\left(\mathrm{kg} / \mathrm{m}^{2}\right)$ was observed maximum in $\mathrm{WH}$ $1021\left(0.53 \mathrm{~kg} / \mathrm{m}^{2}\right)$ followed by PBW 550 $\left(0.48 \mathrm{~kg} / \mathrm{m}^{2}\right)$ and HD $3059\left(0.43 \mathrm{~kg} / \mathrm{m}^{2}\right)$. Lowest grain yield was in $\mathrm{K} 0307(0.16$ $\left.\mathrm{kg} / \mathrm{m}^{2}\right)$ followed by WH $711\left(0.21 \mathrm{~kg} / \mathrm{m}^{2}\right)$, HD $2967\left(0.22 \mathrm{~kg} / \mathrm{m}^{2}\right)$ and DBW $621(0.24$ $\mathrm{kg} / \mathrm{m}^{2}$ ). Maximum 1000 grain weight was observed in WH 1021 (40.5 g) followed by DBW 90 (39.9 g) and HD 3059 (37.7 g). Minimum 1000 grain weight was found in $\mathrm{K}$ 
0307 (18.5 g) followed by HD 2967 (22.5 g), WH 711 (25.5) and DBW 621 (27.4 g). Maximum harvest index (\%) was found in HD 3059 (37.30 \%) followed by HD 2967 (36.70 \%), PBW 550 (36.70 \%), PBW 373 $(36.30 \%)$ and WH $1105 \quad(35.30 \%)$. Minimum harvest index was in HD 2851 (33.30\%) followed by K 0307 (33.70 \%), WH 711 (34.00\%), WH $1124(34.30 \%)$ and HD 2733 (34.30\%).

In stress condition plants produce various protective cellular compounds. The results of the present study are in accordance with the results of Usha and Bhumika (2012) who reported that the overall total antioxidant activity of wheat varieties increase under of stress. Mohammed and Tarpley (2009) showed that susceptible genotypes have low antioxidant activity as compare to tolerant at different growth stages. Thermo tolerance capacity was analyzed using total antioxidant capacity parameter and was found high in thermo tolerant genotypes as compared to thermo susceptible genotypes Kumar et al., (2013). Similar results were found by Almeselmani et al., (2006); (Asthir 2015). Membrane is the first line of defense having many heat responsive sensors which helps plant to trigger its defense mechanism against heat stress, so the integrity of the membrane is an important parameter for heat tolerance Kumar et al., (2012).

The results observed in present study are strongly supported by earlier investigation by Kumar et al., (2013) who observed decrease in the cell membrane stability (CMS) at different stages of growth. Thermo tolerant cultivar showed the highest cell membrane stability index. Lipid peroxidation is considered as one of the most damaging processes known to occur in every living organism. Malondialdehyde (MDA) is produced when polyunsaturated fatty acids in the membrane undergone lipid peroxidation.
MDA content reflects the degree of damage at adverse conditions and is an indicator of lipid peroxidation. Our findings are in accordance with Sairam et al., (2000) reported lowest MDA content in tolerant genotype and highest MDA content in susceptible genotype of wheat under high temperature stress conditions. Mahla et al., (2011) also found more MDA content in heat susceptible cultivar then in a heat tolerant cultivar. Carotenoids are acknowledged for protection in cellular structure from oxidative damage, which stabilizes the membrane fluidity Wahid et al., (2007). Biomass $\left(\mathrm{kg} / \mathrm{m}^{2}\right)$, grain yield $\left(\mathrm{kg} / \mathrm{m}^{2}\right), 1000$ grain weight $(\mathrm{g})$ and harvest Index (\%) were high in heat tolerant genotypes as compare to heat sensitive genotypes as indicated in Table 3 . The results are corroborated with the results of many investigators Dhyani et al., (2013); Saxena et al., (2016); Dwivedi et al., (2017).

The selection of wheat genotypes with better grain yield and heat tolerance at reproductive stage is the principal aim of wheat production. In this study, late sown condition caused significant changes in total antioxidant activity (TAA), cell membrane stability (CMS), lipid peroxidation in term of MDA content, carotenoids content and yield attributes in different wheat genotype studied. Significant reductions in CMS, Carotenoids content and increase in TAA and lipid peroxidation-MDA content from anthesis to 14DAA under terminal heat stress conditions were observed. The protective role of TAA and carotenoids content was evident in tolerant genotypes associated with cell membrane stability and stable yield under terminal heat stress condition. This study concludes that the wheat WH 1021 was found as tolerant genotypes as it is least affected by heat stress and found to differ in their ability to respond which could be useful to develop wheat tolerant varieties in breeding programs. 


\section{References}

Almeselmani, M., Deshmukh, P. S., and Sairam, R. K. 2009. High temperature stress tolerance in wheat genotypes: Role of antioxidant defence enzymes. Acta Agronomica Hungarica. 57: 114.

Almeselmani., M, Deshmukh., PS, Sairam., RK, Kushwaha., SR and Singh, TP. 2006. Protective role of antioxidant enzymes under high temperature stress. Plant Sciences. 171:382-388.

Anonymous (2015).Indian institute of Wheat and Barley Research, Karnal, Progress Report 2014-15.

Asada, K., 2006. Production and scavenging of reactive oxygen species in chloroplasts and their functions. Plant Physiology. 141(2): 391-396.

Asthir, B., 2015. Protective mechanisms of heat tolerance in crop plants. Journal of Plant Interactions. 10: 202-210.

Dhyani, K., Ansari, M.W., Rao, Y.R., Verma, R.S., Shukla, A. and Tuteja, N. 2013.Comparative physiological response of wheat genotypes under terminal heat stress. Plant Signaling and Behavior.8: 245-264.

Dwivedi, R., Prasad, S., Jaiswal, B., Kumar, A. and Tiwari, A. 2017. Evaluation of wheat genotypes (Triticum aestivum L .) at Grain Filling Stage for heat tolerance. International Journal of Pure and Applied Bioscience. 5: 971-975.

Heath, R.L., and Packer, L.1968. Photoperoxidation in isolated chloroplasts. I. Kinetics and stoichiometry of fatty acid peroxidation. Archieves of Biochemistry and Biophysics. 125: 189-198.

Hijioka, Y., Lin, E., Pereira, J. J., Corlett, R. T., Cui, X., and Insarov, G. E. 2014. In V. R. Barros, C. B. Field, D. J. Dokken, M. D. Mastrandrea, K. J.
Mach, T. E. Bilir, M. Chatterjee, K. L. Ebi, Y. O. Estrada, R. C. Genova, B. Girma, E. S. Kissel, A. N. Levy, S. MacCracken, P. R. Mastrandrea, and L. L. White (Eds.), Climate Change 2014: Impacts, Adaptation, and Vulnerability. Part B: Regional Aspects. Contribution of Working Group II to the Fifth Assessment Report of the Intergovernmental Panel on Climate Change (pp. 1327-1370). Cambridge, United Kingdom and New York, NY, USA: Cambridge University Press.

Ibrahim, A.M.H., and Quick, J.S. 2001. Heritability of heat tolerance in winter and spring wheat. Crop Science.41:1401-1405.

Karin, H., Michael, T., Astrid, W., Gerhard, S., Alexander, S. and Dieter, G. 2002. Complex interactive effects of drought and ozone stress on the antioxidant defence systems of two wheat cultivars. Plant Physiology and Biochemistry. 40: 691-696.

Kumar, S., Kumari, P., Kumar, U., Grover, M., Singh, A.K., Singh, R. and Sengar, R.S. 2013. Molecular approaches for designing heat tolerant wheat. Journal of Plant Biochemistry and Biotechnology. 22: 359-371.

Kumar., RR, Goswami. S, Sharma., SK, Singh., K, Gadpayle., KA, Kumar., N, Rai., GK, Singh., $M$ and Rai, R.D 2012. Protection against heat stress in wheat involves change in cell membrane stability, antioxidant enzymes, osmolyte, $\mathrm{H}_{2} \mathrm{O}_{2}$ and transcript of heat shock protein. International Journal of Plant Physiology and Biochemistry. 4(4):8391.

Mahla, R., Madan, S., Rana, M.K. and Munjal, R. 2011. Antioxidant Defense System in Wheat (Triticum aestivum L.) Seedlings under Heat Stress and 
Revival Conditions. Journal of Agricultural Science and Technology. 5(1):27-34.

Mishra, D., Shekhar, S., Agrawal, L., Chakraborty, S. and Chakraborty, N. 2017. Cultivar-specific high temperature stress responses in bread wheat (Triticum aestivum L.) associated with physicochemical traits and defense pathways. Food Chemistry. 221:1077-1087.

Mishra, V., Srivastava, G., Prasad, S. M and G. Abraham 2008. Growth, photosynthetic pigments and photosynthetic activity during seedling stage of cowpea (Vigna unguiculata) in response to UV-B and dimethoate. Pesticide Biochemistry and Physiology. 92.

Mittler, R., 2002. Oxidative stress, antioxidants and stress tolerance. Trends Plant Scicences. 7(9):405-410.

Mohammed, A.R., and Tarpley, L. 2009. Impact of high night time temperature on respiration, membrane stability, antioxidant capacity, and yield of rice plants. Crop Science.49:313-322.

Prieto, P., Pineda, M. and Agilar, M. 1999. Spectrophotometric quantification of antioxidant capacity through the formation of a phosphor molybdenum complex: specific application to the determination of vitamin E. Analytical Biochemistry. 269: 337-341.

Rao, A.R., and Whitcombe, J.R. 1977.Genetic adaptation for vernalization requirement in Napalese Raowheat and barley. Annals of Applied Biology. 85: 121-130.
Sairam, R.K., Srivastava, G.C. and Saxena, D.C. 2000. Increased antioxidant activity under elevated temperatures: a mechanism of heat stress tolerance in wheat genotypes. Biologia Plantarum. 43: 245-251.

Saxena, D.C., Sai Prasad, S.V., Parashar, R. and Rathi, I. 2016 Phenotypic characterization of specific adaptive physiological traits for heat tolerance in wheat. Indian Journal of Plant Physiology. 21:318-322.

Slafer, G.A., and Rawson, H. 1994.Sensitivity of wheat phasic development to major environmental factors: a reexamination of some assumptions made by physiologists and modelers. Australian Journal of Plant Physiology. 21:393-426.

Sullivan, C.Y., 1972.Mechanisms of heat and drought resistance in grain sorghum and methods of measurements. In: Rao, N.G.O. and. House, L.R. (eds.), "Sorghum in seventies". Oxford and IBH, ND, pp. 112-120.

Usha, C., and Bhumika, P. 2012. Oxidative stress in five wheat varieties (Triticum aestivum L.) exposed to water stress and study of their antioxidant enzyme defence system, water stress responsive metabolites and $\mathrm{H}_{2} \mathrm{O}_{2}$ accumulation. Brazalian Journal of Plant Physiology.24: 117-130.

Wahid, A.S., Gelani, M. Ashraf, and M.R, Foolad. 2007. Heat tolerance in plants: An soverview. Environmental and Experimental Botany. 61:199-223.

\section{How to cite this article:}

Pooja, Renu Munjal and Kirpa Ram. 2017. Evaluation of Wheat Genotypes for Protective Mechanisms of Terminal Heat Stress. Int.J.Curr.Microbiol.App.Sci. 6(11): 2623-2633. doi: https://doi.org/10.20546/ijcmas.2017.611.308 